\title{
Article \\ Clinical Breakpoint of Apramycin to Swine Salmonella and Its Effect on Ileum Flora
}

\author{
Xinyu Dai ${ }^{1,2,+}$, Yufeng Gu ${ }^{1,2,+}$, Jinli Guo ${ }^{1,2}$, Lingli Huang ${ }^{1,2}$, Guyue Cheng ${ }^{1,2}$, Dapeng Peng ${ }^{1,2, *}$ \\ and Haihong Hao ${ }^{1,2, *}$
}

1 National Reference Laboratory of Veterinary Drug Residues and MOA Key Laboratory for Detection of Veterinary Drug Residues, Huazhong Agricultural University, Wuhan 430070, China; 15207157890@163.com (X.D.); guyufeng@webmail.hzau.edu.cn (Y.G.); guoj1@personalbio.cn (J.G.); huanglingli@mail.hzau.edu.cn (L.H.); chengguyue@mail.hzau.edu.cn (G.C.)

2 MOA Laboratory for Risk Assessment of Quality and Safety of Livestock and Poultry Products, Huazhong Agricultural University, Wuhan 430070, China

* Correspondence: pengdapeng@mail.hzau.edu.cn (D.P.); haihong_hao@aliyun.com (H.H.); Tel.: +86-027-87287140 (ext. 8115) (H.H.)

+ These authors contributed equally to this work.

check for updates

Citation: Dai, X.; Gu, Y.; Guo, J.; Huang, L.; Cheng, G.; Peng, D.; Hao, H. Clinical Breakpoint of Apramycin to Swine Salmonella and Its Effect on Ileum Flora. Int. J. Mol. Sci. 2022, 23, 1424. https://doi.org/10.3390/ ijms23031424

Academic Editor: Cidália Pina-Vaz

Received: 11 November 2021

Accepted: 21 December 2021

Published: 26 January 2022

Publisher's Note: MDPI stays neutral with regard to jurisdictional claims in published maps and institutional affiliations.

Copyright: (C) 2022 by the authors. Licensee MDPI, Basel, Switzerland. This article is an open access article distributed under the terms and conditions of the Creative Commons Attribution (CC BY) license (https:// creativecommons.org/licenses/by/ $4.0 /)$.

\begin{abstract}
The purpose of this study was to establish the clinical breakpoint (CBP) of apramycin (APR) against Salmonella in swine and evaluate its effect on intestinal microbiota. The CBP was established based on three cutoff values of wild-type cutoff value $\left(\mathrm{CO}_{\mathrm{WT}}\right)$, pharmacokinetic-pharmadynamic $(\mathrm{PK} / \mathrm{PD})$ cutoff value $\left(\mathrm{CO}_{\mathrm{PD}}\right)$ and clinical cutoff value $\left(\mathrm{CO}_{\mathrm{CL}}\right)$. The effect of the optimized dose regimen based on ex vivo PK/PD study. The evolution of the ileum flora was determined by the 16rRNA gene sequencing and bioinformatics. This study firstly established the $\mathrm{CO}_{\mathrm{WT}}, \mathrm{CO}_{\mathrm{PD}}$ in ileum, and $\mathrm{CO}_{\mathrm{CL}}$ of APR against swine Salmonella, the value of these cutoffs were $32 \mu \mathrm{g} / \mathrm{mL}, 32 \mu \mathrm{g} / \mathrm{mL}$ and $8 \mu \mathrm{g} / \mathrm{mL}$, respectively. According to the guiding principle of the Clinical Laboratory Standards Institute (CLSI), the final CBP in ileum was $32 \mu \mathrm{g} / \mathrm{mL}$. Our results revealed the main evolution route in the composition of ileum microbiota of diarrheic piglets treated by APR. The change of the abundances of Bacteroidetes and Euryarchaeota was the most obvious during the evolution process. Methanobrevibacter, Prevotella, S24-7 and Ruminococcaceae were obtained as the highest abundance genus. The abundance of Methanobrevibacter increased significantly when APR treatment carried and decreased in cure and withdrawal period groups. The abundance of Prevotella in the tested groups was significantly lower than that in the healthy group. A decreased of abundance in S24-7 was observed after Salmonella infection and increased slightly after cure. Ruminococcaceae increased significantly after Salmonella infection and decreased significantly after APR treatment. In addition, the genera of Methanobrevibacter and Prevotella were defined as the key node. Valine, leucine and isoleucine biosynthesis, D-Glutamine and D-glutamate metabolism, D-Alanine metabolism, Peptidoglycan and amino acids biosynthesis were the top five Kyoto Encyclopedia of Genes and Genomes (KEGG) pathways in the ileum microbiota of piglets during the Salmonella infection and APR treatment process. Our study extended the understanding of dynamic shift of gut microbes during diarrheic piglets treated by APR.
\end{abstract}

Keywords: Salmonella; apramycin; wild-type cutoff value; PK/PD cutoff value; clinical cutoff value; clinical breakpoint; $16 S$ rRNA gene sequencing

\section{Introduction}

Salmonellosis is one of the most frequent foodborne zoonotic diseases, characterised by abdominal cramps, diarrhoea, fever and vomiting, constituting a worldwide major public health concern [1]. Breeding sows asymptomatically infected with Salmonella can be naturally transmitted directly or indirectly between animals and humans, for example through the consumption of contaminated animal derived food or through contact with 
infected animals [2]. The aminoglycoside antibiotics interfered with translational fidelity by binding to the $30 \mathrm{~S}$ ribosomal subunit, exhibiting excellent characteristics as broad-spectrum antibacterial agents with desirable bactericidal activity against Gram-negative bacteria and mycobacteria [3,4]. Apramycin (APR), equipping a bicyclic sugar moiety with a monosubstituted deoxystreptamine, is distinct from other aminoglycosides [5]. This distinct structure protects APR from degradation by aminoglycoside modifying enzymes that confer resistance to other aminoglycosides and contributes to offer higher selectivity for bacteria [6]. As a veterinary aminocyclitol aminoglycoside, APR was often used to treat or prevent infections caused by Gram-negative bacteria such as Colibacillosis, Salmonellosis and Enteritis in farm animals $[7,8]$.

Epidemiological investigations of APR-resistant bacteria from foodborne animals demonstrated that prevalence of APR resistance is different among animals [9]. Cross resistance was also observed between APR and other antibiotics such as gentamicin $[10,11]$. However, there is no clinical breakpoint (CBP) to interpret APR against swine Salmonella making it impossible to interpret the results of an antimicrobial susceptibility testing (AST). CBPs are minimum inhibitory concentration (MIC) cutoff values used by diagnostic laboratories to distinguish bacteria as susceptible (S), intermediate (I) or resistant (R) to an antimicrobial agent [12]. The CBP of an antibiotic is established according to defined rules, including the wild-type cutoff values $\left(\mathrm{CO}_{\mathrm{WT}}\right)$ of the organism against the antimicrobial, pharmacokinetic-pharmacodynamics (PK/PD) considerations through the PK/PD cutoff values $\left(\mathrm{CO}_{\mathrm{PD}}\right)$, and clinical cutoff values $\left(\mathrm{CO}_{\mathrm{CL}}\right)$ based on clinical efficacy data $[12,13]$. Comprehensive procedures on setting CBPs for veterinary medicine have been established and documented in the Clinical Laboratory Standards Institute (CLSI)/Veterinary Antimicrobial Susceptibility Testing (VAST) profiles [14].

A highly diversified community of microorganisms parasitize in the mammalian gastrointestinal tract with approximately $10^{12}$ microbes containing about 500-1000 species [15]. The gastrointestinal microbiome is an enormous and dynamic ecosystem, which have a profound impact on health and disease participating in the absorption of nutrients and the expulsion of metabolic wastes from the host and shaping a barrier against the pathogens [16]. For swine, microbiota also contributes to the development of the gastrointestinal immune system and plays a considerable role in diarrhea [17]. Previous study has utilized high-throughput sequencing of the $16 S \mathrm{rRNA}$ gene to characterize gut microbiota of diarrheic piglets [18]. However, gut microbiota evolution track and their correlation to treatment of piglet diarrhea with APR being poorly understood.

The aim of the current investigation was to decide the $\mathrm{CO}_{\mathrm{WT}}, \mathrm{CO}_{\mathrm{PD}}$ and $\mathrm{CO}_{\mathrm{CL}}$ values for the establishment of the CBP and to evaluate the clinical efficacy of APR against swine Salmonella. Then the intestinal flora changes in the composition and evolution of the piglets in healthy phase, Salmonella challenge phase, APR treatment phase, APR cure phase and withdrawal phase was compared and analyzed to identify hub differences in the gastrointestinal tract microbiota of piglets to reveal diarrhea-related bacteria.

\section{Results}

\subsection{MIC Distribution and $\mathrm{CO}_{W T}$}

MICs of APR against 185 swine Salmonella isolates were shown in Table S1. The MICs was distributed in a normal distribution pattern with a definite peak at $8 \mu \mathrm{g} / \mathrm{mL}$. The corresponding $\mathrm{MIC}_{50}$ and $\mathrm{MIC}_{90}$ were determined to be 8 and $16 \mu \mathrm{g} / \mathrm{mL}$, respectively. The fitted MIC distribution, encompassing 95\% of the wild-type isolates, was processed in Ecoffinder software and the epidemiological cutoff (ECOFF) value was therefore calculated to be $32 \mu \mathrm{g} / \mathrm{mL}$ (Figure 1). 


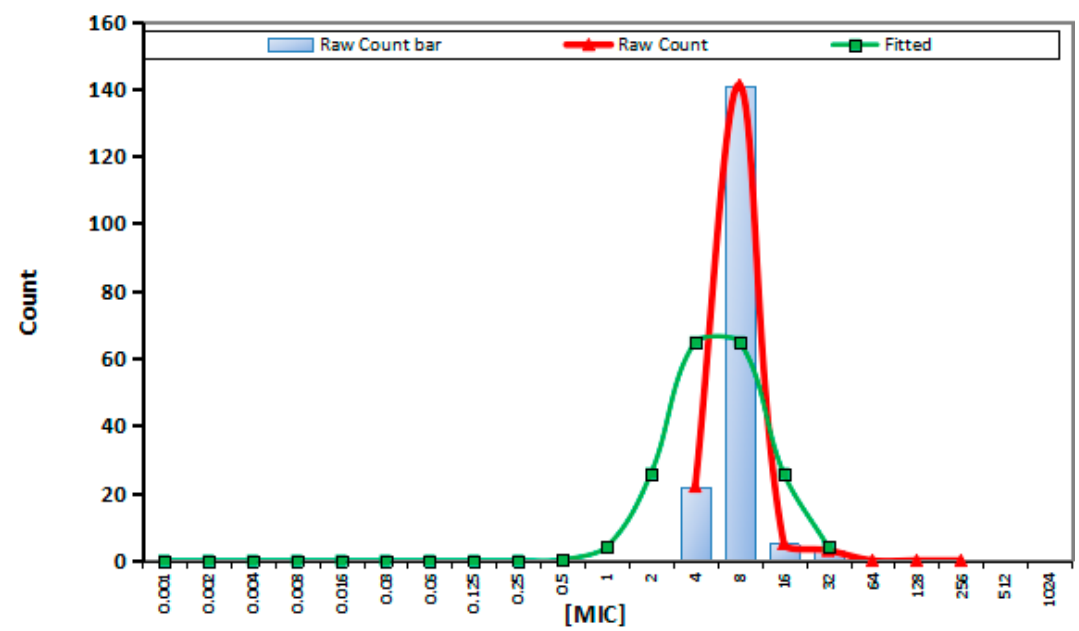

Figure 1. Nonlinear regression of MIC distribution for APR against swine Salmonella.

\subsection{PD Study of APR against Swine Salmonella Strain 10\# In Vitro and Ex Vivo}

The MIC and minimum bactericidal concentration (MBC) values of APR against swine Salmonella 10\# were 8 and $16 \mu \mathrm{g} / \mathrm{mL}$ both in Mueller-Hinton (MH) broth and ileum fluid. The mutant prevention concentration (MPC) of APR against swine Salmonella 10\# was $51.2 \mu \mathrm{g} / \mathrm{mL}$. The Post-antibiotic effect (PAE) of swine Salmonella exposed to APR for $1 \mathrm{~h}$ and $2 \mathrm{~h}$ were ranging from $0.51-1.42 \mathrm{~h}, 0.45-6 \mathrm{~h}$, respectively (Table S2). The in vitro time-killing curves showed that APR displayed a concentration-dependent bactericidal effect as increasing drug concentrations induced more rapid and radical killing effects (Figure 2A). Sustained growth inhibition was observed only within the initial $6 \mathrm{~h}$ in the $\mathrm{MH}$ broth containing lower than $2 \times$ MIC concentration of APR. When 10\# was exposed to APR at a concentration equal to or higher than $2 \times \mathrm{MIC}$, the total bacteria were significantly decreased to the undetectable level $(\leq 10 \mathrm{CFU} / \mathrm{mL})$ after $24 \mathrm{~h}$ of APR exposure. As presented in Figure 2B,C, the bacteria were drastically reduced to the undetectable limit $(<10 \mathrm{CFU} / \mathrm{mL}$ ) after exposure to the ileum fluid collected between $2-10 \mathrm{~h}$ after oral administration, suggesting that APR exhibited a concentration-dependent killing mechanism in the ex vivo environment, consistent with the in vitro bactericidal effect. Slight regrowth was observed in the ileum fluid samples collected at 1 and $12 \mathrm{~h}$ after incubation for $6 \mathrm{~h}$.

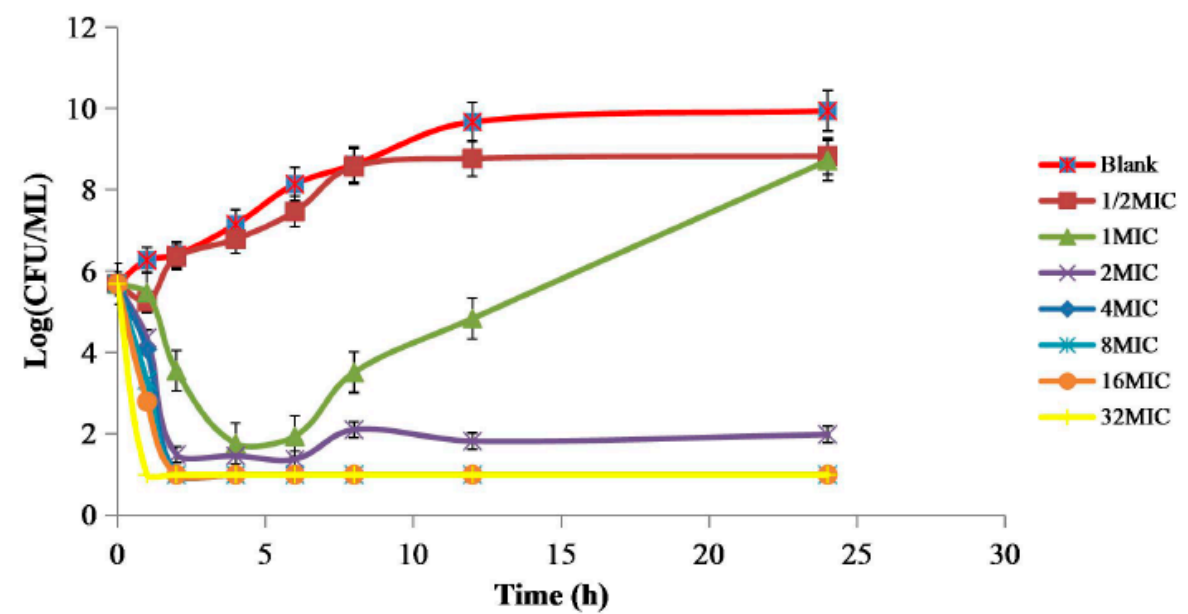

(A)

Figure 2. Cont. 


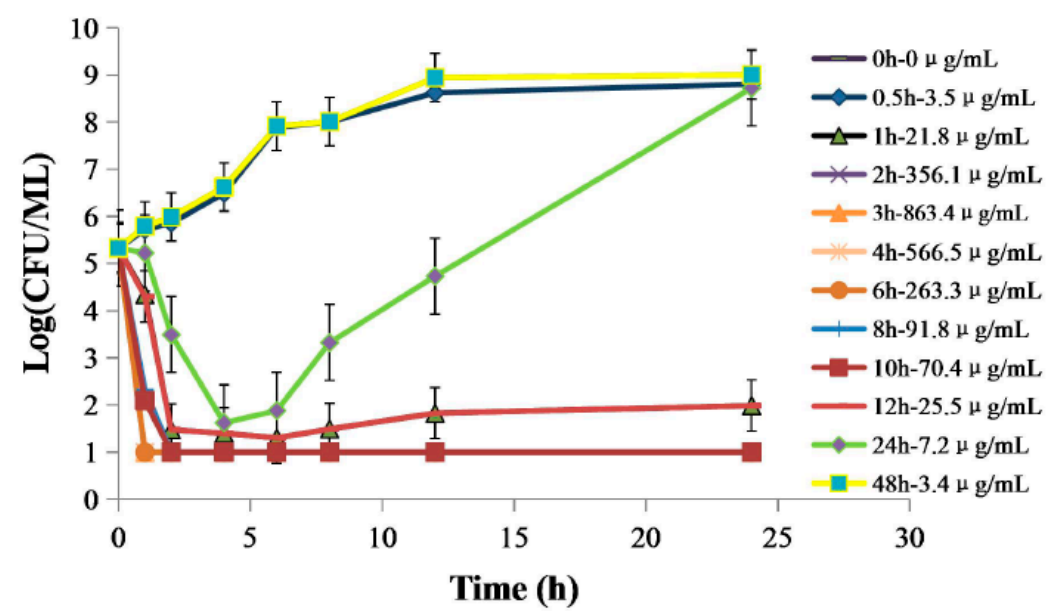

(B)

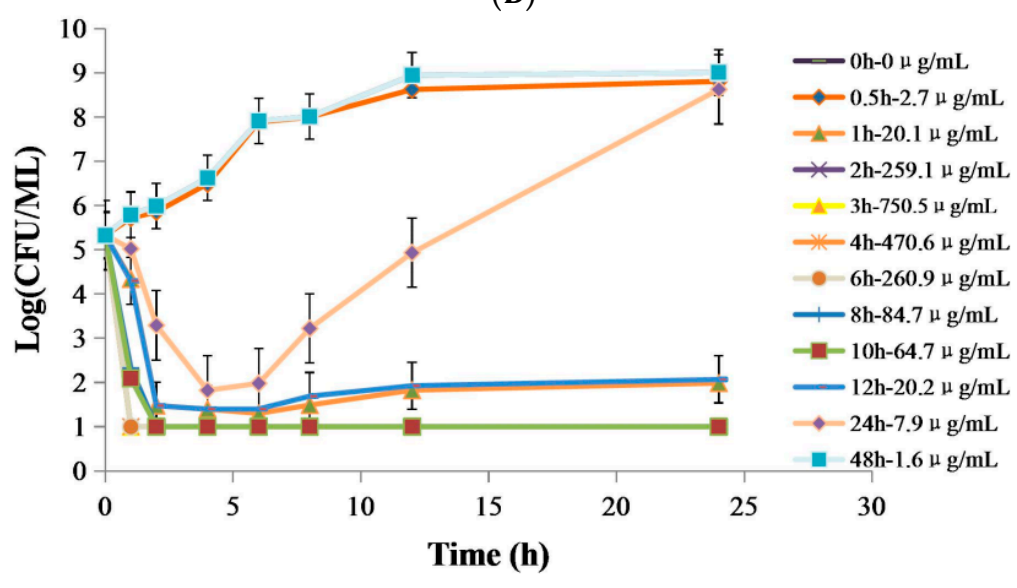

(C)

Figure 2. The time-killing curve of APR against swine Salmonella in vitro and ex vivo. (A) The time-killing curve in $\mathrm{MH}$ broth; (B) the time-killing curve in ileum fluid of health piglets; (C) the time-killing curve in ileum fluid of diarrhea piglets.

\subsection{PK/PD Analysis in the Plasma and Ileum Fluid and Establishment of $C \mathrm{CO}_{P D}$}

Single-dose PK of APR in plasma and ileum contents was shown in Figure 3. Concentrations of APR in plasma and ileum contents at a series of time points were shown in Tables S3 and S4. Remarkable gap of drug concentrations was observed between plasma and ileum. The absorption of APR after oral administration was limited, but the concentration in the ileum fluid was markedly higher compared to the APR concentration in plasma (Table 1). The $\mathrm{AUC}_{0-24 \mathrm{~h}}$ value in ileal contents was also significantly higher than that in plasma. The $\mathrm{C}_{\max }$ in plasma was far lower than the MIC of the selected strain 10\#, so the APR could not achieve the purpose of treatment. Therefore, the PK parameters of ileal contents were selected for PK/PD analysis.

The relationship between the antimicrobial efficacy and the ex vivo PK/PD parameter of $\mathrm{AUC}_{0-24 \mathrm{~h}} / \mathrm{MIC}$ ratios was fitted by using the inhibitory sigmoid $\mathrm{E}_{\max }$ model. The model parameters, including the Hill coefficient $(\mathrm{N}), \mathrm{E}_{0}, \mathrm{E}_{\max }$ and $\mathrm{AUC}_{0-24 \mathrm{~h}} / \mathrm{MIC}$ values for the three levels of growth inhibition were presented in Table 2; the $\mathrm{AUC}_{0-24 \mathrm{~h}} / \mathrm{MIC}$ values for bacteriostatic activity $(\mathrm{E}=0)$, bactericidal activity $(\mathrm{E}=-3)$ and a virtual elimination effect $(\mathrm{E}=-4)$ were $46.65,48.90$ and $49.98 \mathrm{~h}$ in healthy piglets, respectively. The $\mathrm{AUC}_{0-24 \mathrm{~h}} / \mathrm{MIC}$ values for bacteriostatic activity $(\mathrm{E}=0)$, bactericidal activity $(\mathrm{E}=-3)$, and a virtual elimination effect $(\mathrm{E}=-4)$ were $46,58.38$ and $65.53 \mathrm{~h}$ in infection piglets, respectively. Based on Monte Carlo Simulation (MCS), the probability of target attainment (PTA) under different MICs was calculated (Table S5). The maximum MIC with a PTA equal to or greater than $90 \%$ was selected as $\mathrm{CO}_{\mathrm{PD}}$. Therefore, the $\mathrm{CO}_{\mathrm{PD}}$ of APR against Salmonella was $32 \mu \mathrm{g} / \mathrm{mL}$. 


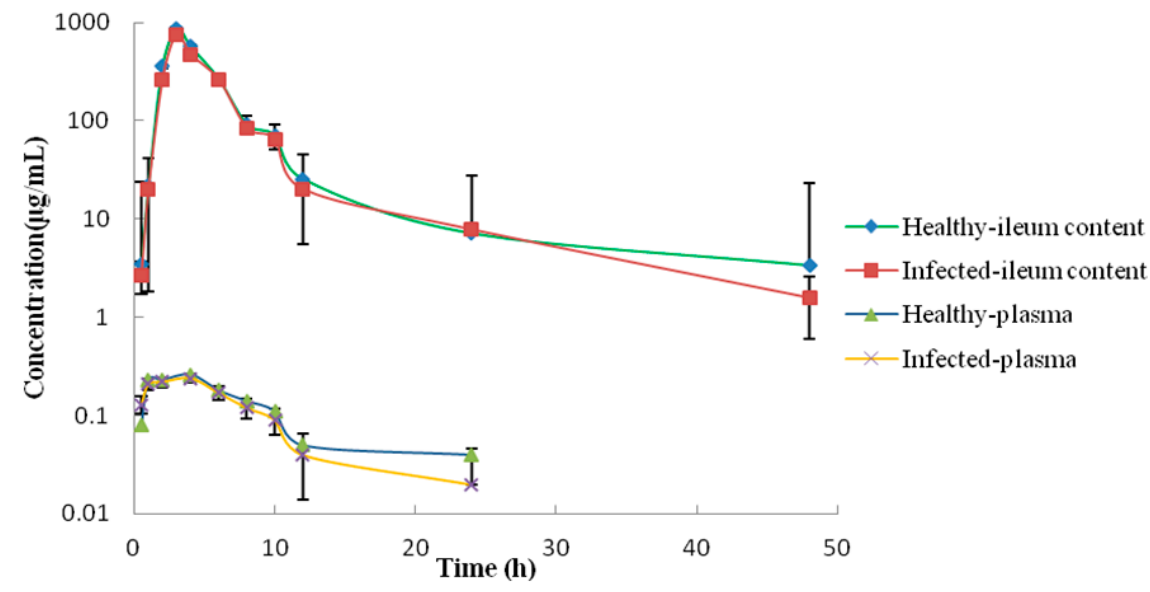

Figure 3. Concentration-time curve of APR in plasma and ileum content of piglets.

Table 1. Integrated PK parameters for APR in plasma and ileum contents after oral administration of $30 \mathrm{mg} / \mathrm{kg}$ b.w.

\begin{tabular}{cccccc}
\hline & & \multicolumn{2}{c}{ Plasma } & \multicolumn{2}{c}{ Ileum Contents } \\
\cline { 3 - 6 } Parameter & Units & $\begin{array}{c}\text { Healthy } \\
\text { Group }\end{array}$ & $\begin{array}{c}\text { Infected } \\
\text { Group }\end{array}$ & Healthy Group & Infected Group \\
\hline $\mathrm{C}_{\max }$ & $\mu \mathrm{g} / \mathrm{mL}$ & $0.26 \pm 0.01$ & $0.24 \pm 0.01$ & $863.4 \pm 18.2$ & $750.5 \pm 24.82$ \\
$\mathrm{AUC}_{0-24 \mathrm{~h}}$ & $\mathrm{~h} \cdot \mu \mathrm{g} / \mathrm{mL}$ & $4.12 \pm 0.76$ & $3.41 \pm 0.48$ & $3287.25 \pm 23.41$ & $2855.83 \pm 19.81$ \\
$\mathrm{AUMC}_{0-24 \mathrm{~h}}$ & $\mathrm{~h}^{2} \cdot \mu \mathrm{g} / \mathrm{mL}$ & $63.68 \pm 2.12$ & $45.13 \pm 1.71$ & $19,965.83 \pm 151.2$ & $17,421.25 \pm 132.5$ \\
$\mathrm{~T}_{\max }$ & $\mathrm{H}$ & $4.0 \pm 0.0$ & $4.0 \pm 0.0$ & $3.0 \pm 0.0$ & $3.0 \pm 0.0$ \\
$\mathrm{~T}_{1 / 2}$ & $\mathrm{H}$ & $20.00 \pm 1.02$ & $15.77 \pm 1.93$ & $8.74 \pm 1.01$ & $7.83 \pm 0.78$ \\
$\mathrm{MRT}_{0-24 \mathrm{~h}}$ & $\mathrm{H}$ & $15.47 \pm 0.92$ & $13.22 \pm 1.02$ & $6.07 \pm 0.24$ & $6.10 \pm 0.42$ \\
$\mathrm{CL}_{\mathrm{N} / \mathrm{F}}$ & $\mathrm{mL} / \mathrm{h} / \mathrm{kg}$ & $5.69 \pm 0.01$ & $7.76 \pm 0.02$ & $9.0 \pm 0.02$ & $10.04 \pm 0.01$ \\
\hline
\end{tabular}

Note: $\mathrm{C}_{\max }=$ maximum concentration, $\mathrm{AUC}_{0-24 \mathrm{~h}}=$ area under concentration curve, $\mathrm{AUMC}_{0-24 \mathrm{~h}}=$ first-order area under concentration curve, $\mathrm{T}_{1 / 2 \lambda}=$ elimination half-life, $\mathrm{T}_{\max }=$ time of maximum concentration, $\mathrm{MRT}_{0-24 \mathrm{~h}}=$ mean residence time, $\mathrm{CL} / \mathrm{F}=$ body clearance scaled by bioavailability.

Table 2. Ex vivo PK parameters of APR against swine Salmonella.

\begin{tabular}{cccc}
\hline \multirow{2}{*}{ Parameter } & Unit & \multicolumn{2}{c}{ Value } \\
\cline { 3 - 4 } & & Healthy Group & Infected Group \\
\hline $\mathrm{E}_{\max }$ & $\log 10 \mathrm{CFU} / \mathrm{mL}$ & 4.25 & 3.48 \\
$\mathrm{E}_{0}$ & $\mathrm{~h}$ & -5.69 & -5.33 \\
$\mathrm{EC}_{50}$ & $\log 10 \mathrm{CFU} / \mathrm{mL}$ & 47.16 & 49.37 \\
$\mathrm{~N}$ & - & 27.25 & 6.10 \\
$\mathrm{E}_{\max }-\mathrm{E}_{0}$ & $\log 10 \mathrm{CFU} / \mathrm{mL}$ & 9.94 & 8.81 \\
$\mathrm{AUC}_{0-24 \mathrm{~h}} / \mathrm{MIC}(\mathrm{E}=0)$ & $\mathrm{h}$ & 46.65 & 46 \\
$\mathrm{AUC}_{0-24 \mathrm{~h}} / \mathrm{MIC}(\mathrm{E}=-3)$ & $\mathrm{h}$ & 48.90 & 58.38 \\
$\mathrm{AUC}_{0-24 \mathrm{~h}} / \mathrm{MIC}(\mathrm{E}=-4)$ & $\mathrm{h}$ & 49.98 & 65.53 \\
\hline
\end{tabular}

\subsection{Dosage Determination and Establishment of $\mathrm{CO}_{C L}$ of APR against Swine Salmonella}

According to the MCS, the ratio of $\mathrm{CL} / \mathrm{f}$ and $\mathrm{AUC}_{0-24 \mathrm{~h}} / \mathrm{MIC}$ under different antibacterial effects were obtained. The doses predicted to obtain bacteriostatic, bactericidal and elimination effects for Salmonella over $24 \mathrm{~h}$ were 4.15, 5.27, $5.91 \mathrm{mg} / \mathrm{kg}$ b.w. Statistics of clinical trial results were shown in Table S6. Then the results of the obtained clinical MIC and Probablity of Cure (POC) were analyzed by the "WindoW" method, and the selection threshold of the $\mathrm{CO}_{\mathrm{CL}}$ was $8-32 \mu \mathrm{g} / \mathrm{mL}$. A $\mathrm{CO}_{\mathrm{CL}}$ value obtained by Correlation and Regression Trees (CART) analysis was to be lower than $12 \mu \mathrm{g} / \mathrm{mL}$. Combining the above two analytical methods, the $\mathrm{CO}_{\mathrm{CL}}$ value was calculated as $8 \mu \mathrm{g} / \mathrm{mL}$. 


\subsection{DNA Sequence Data and Bacterial Community Structure}

Based on $97 \%$ species similarity, all the sequences were clustered into operational taxonomic units (OTUs) with the size ranging from 1570 to 2638 (Table S7). The rarefaction curves (Figure S1) showed that the sequencing depth was near saturation and that the sequencing data included most of the 16S rRNA gene information. A total of 8381 OTUs were identified from all ileum content samples, with 420 of those existing in each group defined as core OTUs (Figure 4). The core OTUs comprised approximately $5 \%$ of the total OTUs while $842,808,800,822$ and 490 OTUs were uniquely identified in the healthy, infection, treatment, cure and withdrawal period group, respectively.

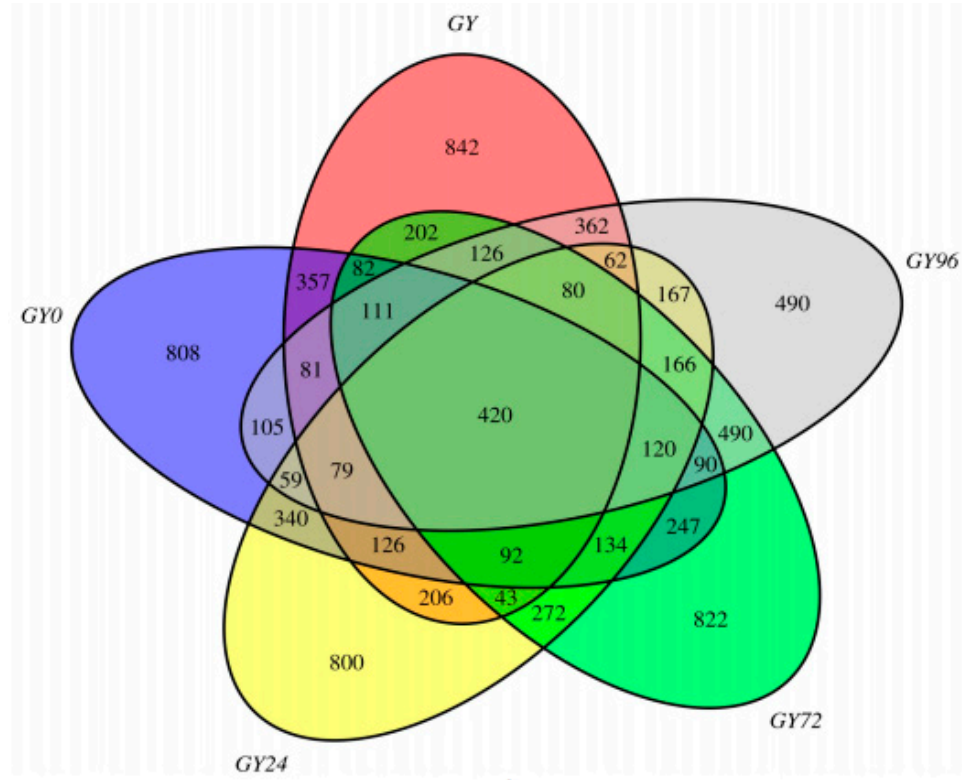

Figure 4. The Venn diagram of the shared and unique OTUs presented in different groups. Note: "GY" represents the healthy group, "GY0" represents the infection group, "GY24" represents the treatment group, "GY72" represents the cure group and "GY96" represents the withdrawal period group.

The microbial complexities in the healthy, infection, treatment, cure and withdraw period groups were demonstrated by Alpha diversity parameters, including richness, Shannon, Simpson, pielou, invsimpson, Chao1, Abundance-based Coverage Estimator (ACE) and good coverage (Figure 5A). The results showed that healthy group samples had the largest alpha-diversity indices followed by those in the cure group, withdrawal period group, infection group and treatment group. To evaluate overall differences in betadiversity, the unweighted pair group method with arithmetic mean clustering (UPGMA), nonmetric multidimensional scaling (NMDS) analysis, the ANOSIM and the principal coordinate analysis (PCoA) was used to identify discrepancies between groups. As shown in Figure 5B, the results of UPGMA and NMDS analysis indicated the clear separation of the bacterial community structure into mainly two large clusters. This difference between the bacterial communities was significant, as determined using the ANOSIM analysis $(\mathrm{R}=0.935, p=0.001)$. PCoA results showed that the ileum microbiota of the healthy group was similar to that of the infection group, and the ileum microbiota of the cure group was similar to that of the withdrawal period. Specifically, the ileum microbiota of the treatment group was distinct to other groups. Collectively, these data point toward the marked difference in the ileum microbiota between these groups. 


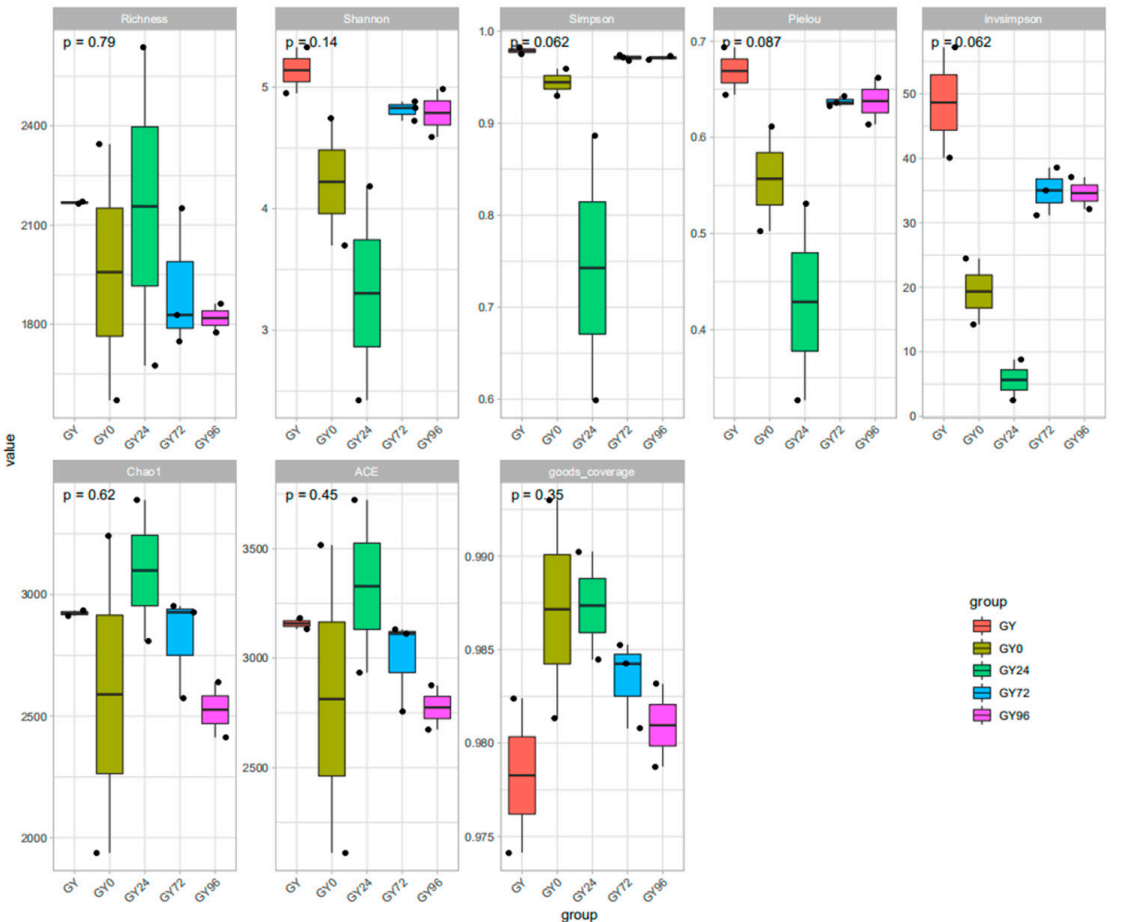

(A)
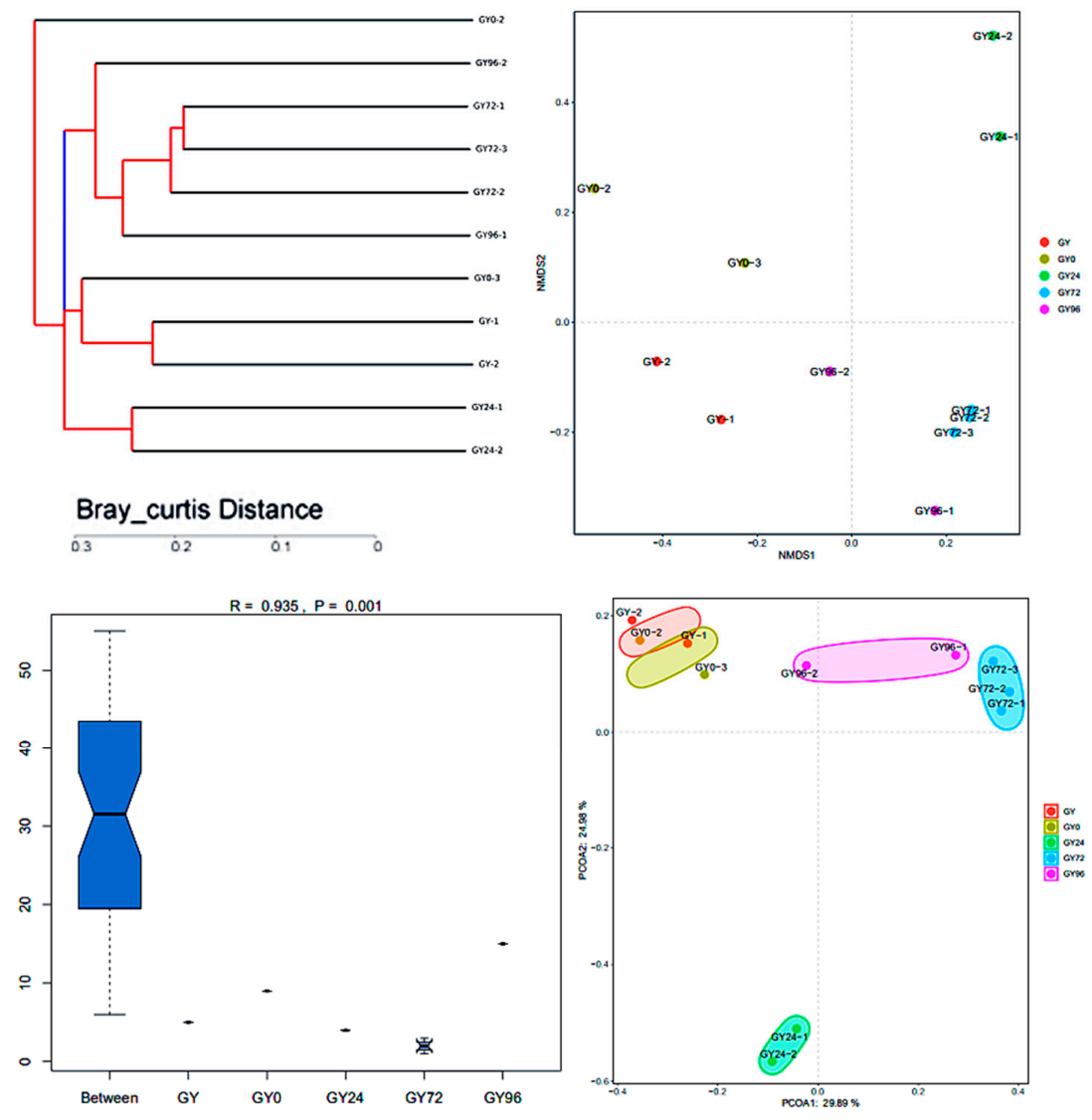

(B)

Figure 5. Alpha and beta diversity parameters presented in the five groups. (A) Alpha diversity parameters of richness, Shannon, Simpson, pielou, invsimpson, Chao1, ACE and good coverage; (B) Beta parameters of UPGMA, NMDS, ANOSIM and PCoA. 
2.6. Analysis of Microbial Flora Composition in Ileum of Piglets under Different Taxonomic Levels

OTUs obtained from samples belonged to 17 phyla, 33 classes, 50 orders, 95 families and 177 genera. Notably, at the phylum level, Firmicutes was the most predominant phylum ranging from $32.23 \%$ to $53.53 \%$. The abundances of Firmicutes were relatively small increase form Salmonella infection to withdrawal period. The Euryarchaeota was significantly increased in the treatment group (from 2.25\% to $47.62 \%$ ) and quickly decreased to the initial level. The shifts of the abundances of Bacteroidetes were precisely opposite to that of the Euryarchaeota and ultimately return to the initial state (Figure 6A). There was also a conspicuous abundances change in the Actinobacteria and Proteobacteria, but no significant differences in other phylum were discerned in these groups. In general, the use of APR in the treatment of piglet diarrhea would not destroy the balance of intestinal flora at the phylum level. Evidently, Prevotellaceae belonging to the phylum Bacteroidetes and Ruminococcace, belonging to the phylum Firmicutes, were the top two abundant families in the ileum. The family S24-7 had significantly lower abundance than Prevotellaceae and Ruminococcace in the healthy group (Figure 6B).

At the genus level, the top four most abundant genera containing 63.25-98.95\% of the total OUTs were Methanobrevibacter, Prevotella, S24-7 and Ruminococcaceae. Methanobrevibacter suddenly increased significantly from APR treatment and decreased slightly in the cure group. The percentage of Prevotella in all other groups was significantly lower than that in the healthy group. A decreased of S24-7 was observed after Salmonella infection and increased slightly after cure. Ruminococcaceae increased significantly after Salmonella infection and decreased significantly after APR treatment (Figure 6B).

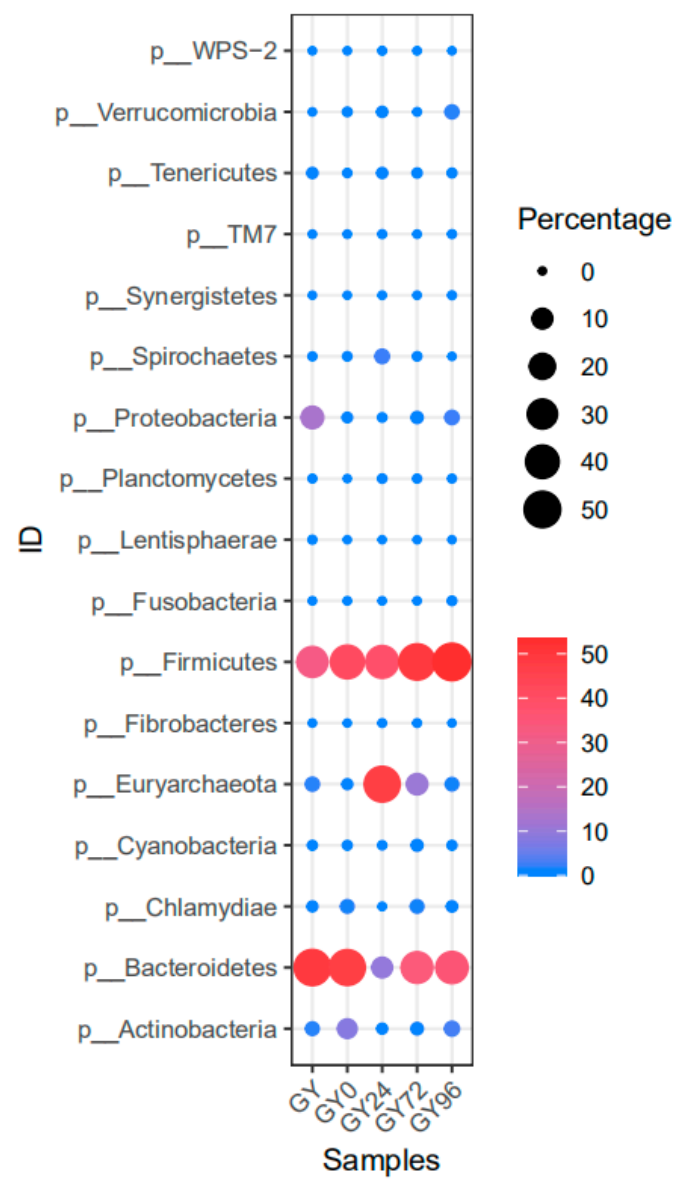

(A)

Figure 6. Cont. 


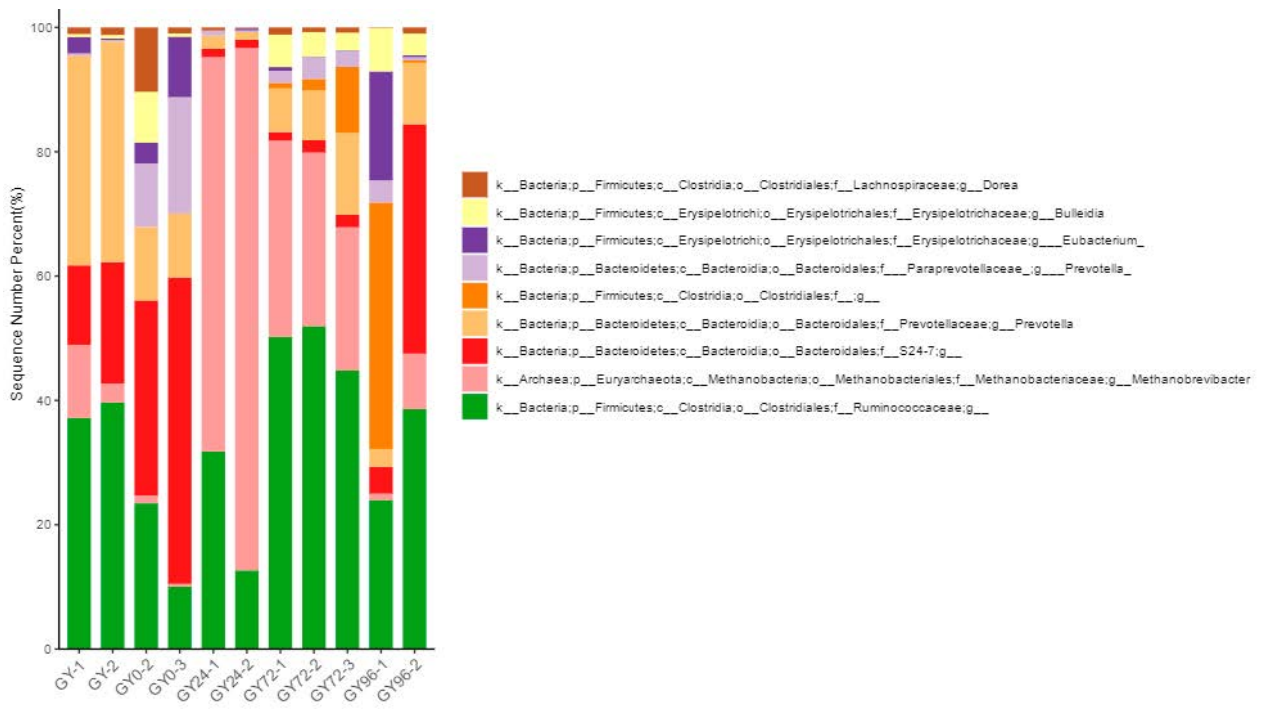

(B)

Figure 6. Taxonomic classification of the $16 S$ rRNA gene sequences at phylum and genus levels. (A) The evolution Flora presented at phylum level; (B) The bacterial community composition at genus level.

Linear Discriminant Analysis Effect Size (LEfSe) analysis was used to identify biomarkers of ileum microbiota of different stages of clinical trial piglets (Figure 7). The differentially abundant phyla detected showed that the Proteobacteria and Lentisphaerae phylum was predominant in the healthy group, while the most abundant genus in the infection group was Pseudobutyrivibrio. It was found that the Leuconostoc was a predominant genus in the cure group, whereas the Eggerthella, Leuconostoc and Sutterella were found to be a dominant genus in withdraw period (Figure 7A). There was an overrepresentation of Proteobacteria and Lentisphaerae, and underrepresentation of other phyla (Figure 7B).

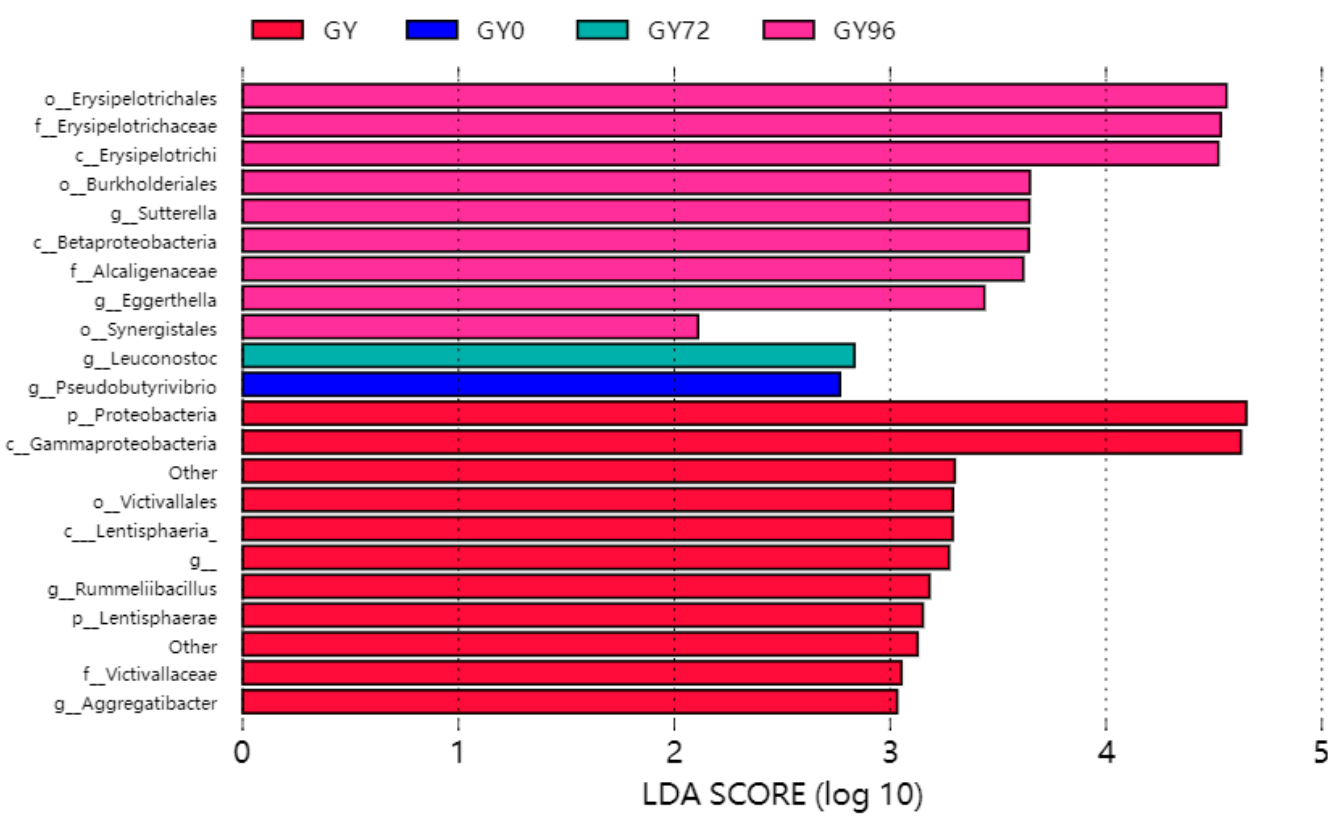

(A)

Figure 7. Cont. 

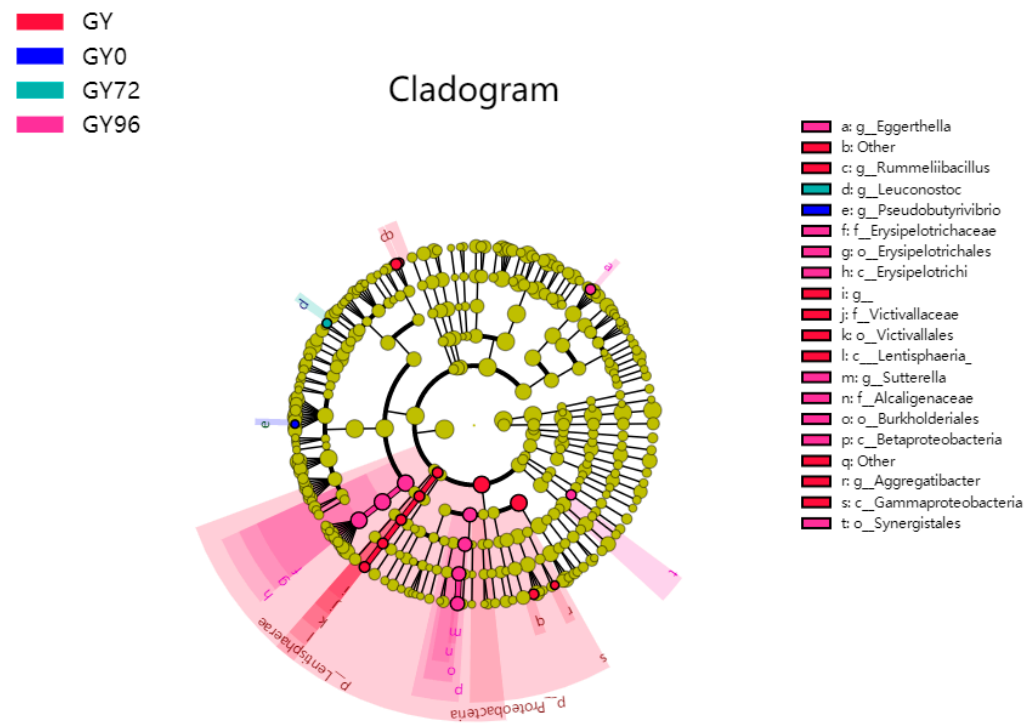

(B)

Figure 7. Different structures of ileum microbiota presented in different groups by the LEfSe analysis. (A) Taxonomic biomarkers found among groups by LEfSe; (B) Cladogram plot of the biomarkers. The size of the node represents the abundance of the taxa. Only taxa with LDA scores $(\log 10)>4$ were shown.

\subsection{Correlations of the Different Dominant Bacterial Communities}

Through Spearman network analysis, the top 30 OTUs with the greatest absolute abundances among all samples were selected for correlation analysis at the genus level. The calculated results were plotted after filtering out differences with a $p$ value greater than 0.05 or a correlation coefficient $\mathrm{R}<0.4$ (Figure 8 ). The top 30 genera were classified into seven phyla, including Actinobacteria, Bacteroidetes, Chlamydiae, Euryarchaeota, Firmicutes, Proteobacteria and Spirochaetes. Interestingly, two genera were found as the core node, including Methanobrevibacter and Prevotella, showed highly correlation to other genus. Hence, the genera Methanobrevibacter and Prevotella might play a vital role in Salmonella infection and APR treatment process.

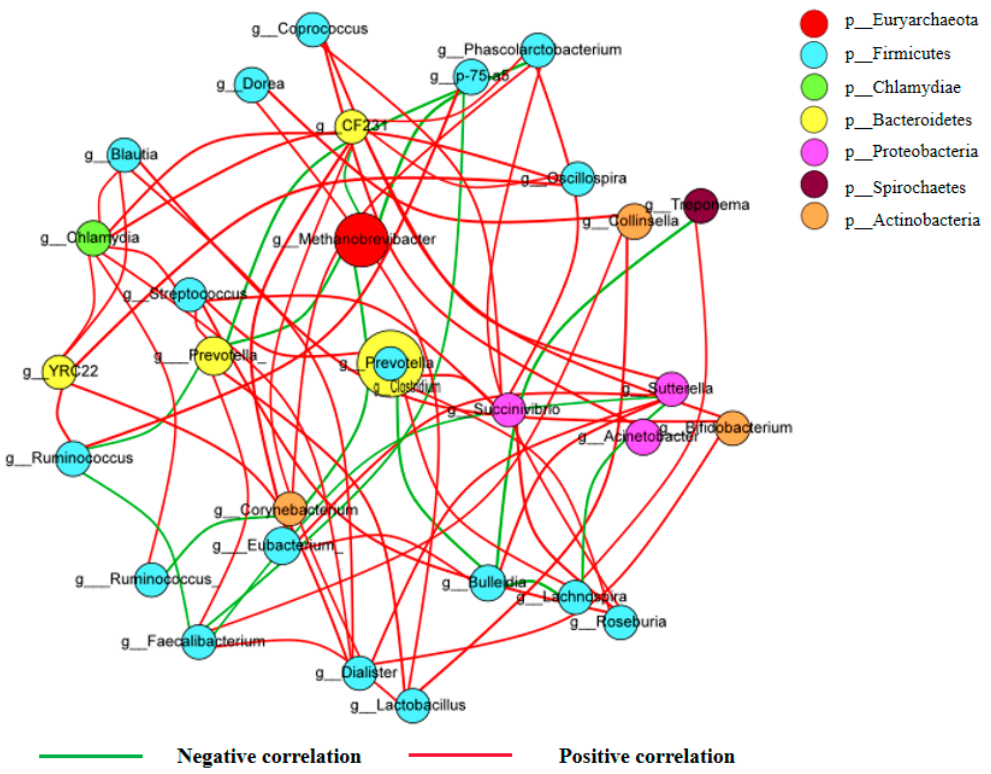

Figure 8. Co-occurrence network existed of ileum flora. 


\subsection{Predicted Kyoto Encyclopedia of Genes and Genomes (KEGG) Pathway}

It was found that KEGG pathways involved in cellular processes, environmental information processing, genetic information processing, human diseases, metabolism and organismal systems were predominant in these groups (Figure 9). Overall, 45 pathways were obtained at level 2, and amino acid metabolism, carbohydrate metabolism, metabolism of cofactors and vitamins, metabolism of other amino acids, biosynthesis of other secondary metabolites, global and overview maps, replication and repair, glycan biosynthesis and metabolism, lipid metabolism and energy metabolism were the top ten KEGG pathways in the ileum microbiota of piglets in this study (Table S8). Moreover, 314 KEGG pathways were found at level 3. Valine, leucine and isoleucine biosynthesis, D-Glutamine and D-glutamate metabolism, D-Alanine metabolism, peptidoglycan biosynthesis and biosynthesis of amino acids were the top five KEGG pathways in the ileum microbiota of piglets.

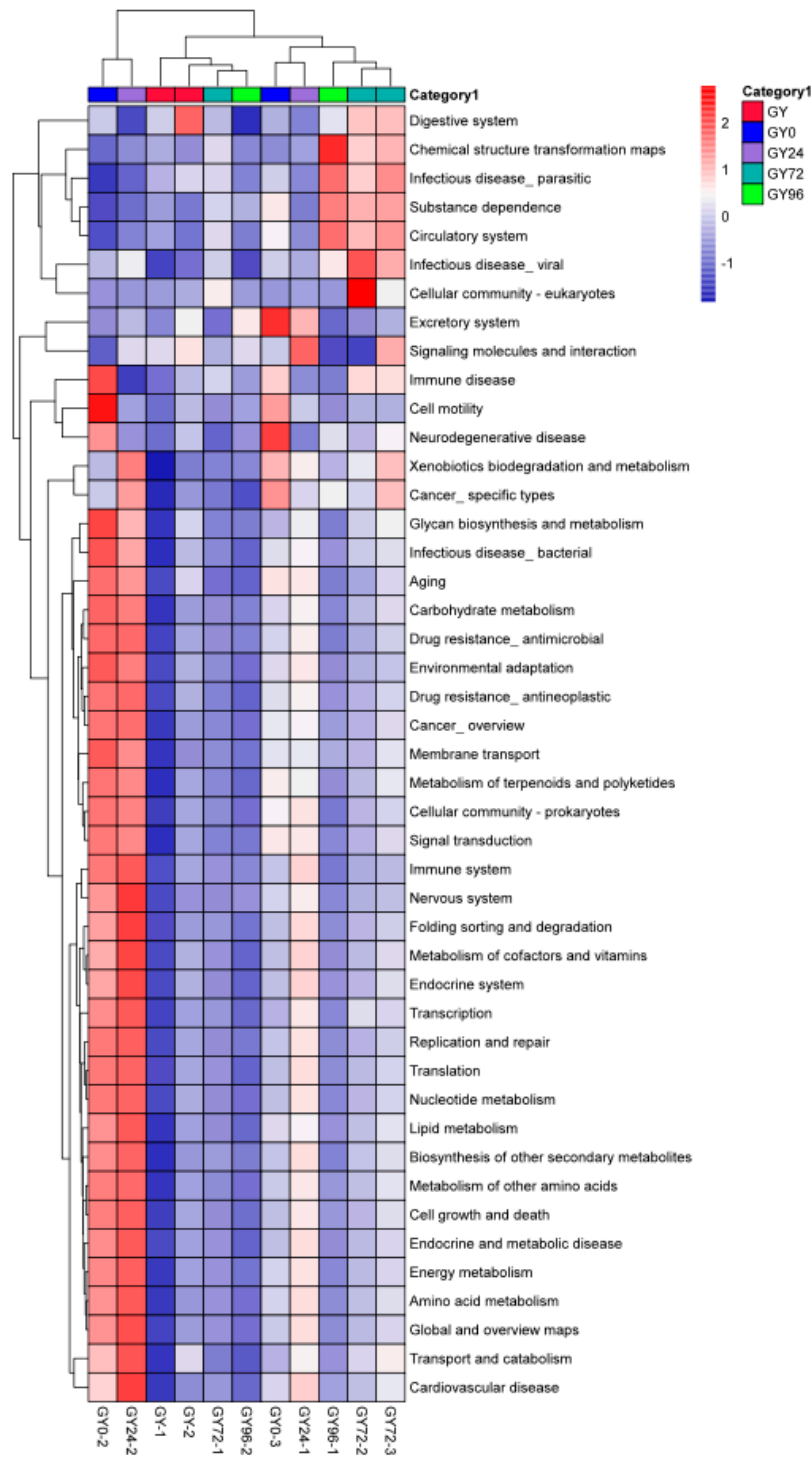

Figure 9. Comparison of variations presented in abundance of known KEGG pathways.

\section{Discussion}

APR is currently used as an orally dosed, non-absorbed veterinary antibiotic to treat diarrheal diseases in poultry and livestock [19]. For APR, the American National Antimicrobial Resistance Monitoring System for animal isolates classed Escherichia coli (E. coli) and Salmonella as resistant if the MICs were higher than $16 \mu \mathrm{g} / \mathrm{mL}$. The MICs were determined for APR of Salmonella $(n=52)$ isolates and the $\mathrm{MIC}_{50}$ and $\mathrm{MIC}_{90}$ values for APR were 4 and 
$8 \mu \mathrm{g} / \mathrm{mL}$, respectively [20]. The $\mathrm{CO}_{\mathrm{WT}}$ for APR of E. coli isolated from chicken intestinal tracts was defined as 16 to $32 \mu \mathrm{g} / \mathrm{mL}$ established by the National Antibiotic Resistance Monitoring Study. It was also reported that the $\mathrm{MIC}_{50}$ and $\mathrm{MIC}_{90}$ for APR against E. coli were 8 and $16 \mu \mathrm{g} / \mathrm{mL}$, and the $\mathrm{CO}_{\mathrm{WT}}$ for APR of E. coli was $16 \mu \mathrm{g} / \mathrm{mL}$ [21]. In this study, the results showed that the $\mathrm{MIC}_{50}$ and $\mathrm{MIC}_{90}$ values for APR against 185 swine Salmonella isolates obtained as 8 and $16 \mathrm{mg} / \mathrm{L}$, and the $\mathrm{CO}_{\mathrm{WT}}$ value for APR of Salmonella was $32 \mu \mathrm{g} / \mathrm{mL}$. Our result was similar to the previous study suggesting that the reliability of our result was reasonably high.

For PK/PD evaluation, tissue distribution should be considered, but the tissue distribution was often overlooked when developing PK/PD targets [22,23]. APR following oral administration with a poor absorption making most of the drugs remained in the gastrointestinal tract, which caused a great difference in the distribution of APR in plasma and intestine. PK studies on APR were mainly based on PK data in plasma. Previous result showed that the peak plasma concentration of APR was $0.23 \mu \mathrm{g} / \mathrm{mL}$ and reached at $2.25 \mathrm{~h}$, the $\mathrm{AUC}_{\mathrm{INF}}$ was $1.59 \mathrm{~h} \cdot \mu \mathrm{g} / \mathrm{mL}$ in plasma after oral administration of $4 \mathrm{mg} / \mathrm{kg}$ [24]. The corresponding $\mathrm{PK}$ parameters of the $\mathrm{C}_{\max }$ and $\mathrm{AUC}_{\mathrm{INF}}$ were lower observed in plasma of pigs and in broiler chickens $[25,26]$. In our study, PK parameters were obtained both in plasma and ileum. The $A U C_{I N F}$ and $C_{\max }$ value in ileal contents were $2855.83 \pm 19.81 \mathrm{~h} \cdot \mu \mathrm{g} / \mathrm{mL}$ and $750.5 \pm 24.82 \mu \mathrm{g} / \mathrm{mL}$, respectively. The $A U C_{I N F}$ and $C_{\max }$ value in plasma were $3.41 \pm 0.48 \mathrm{~h} \cdot \mu \mathrm{g} / \mathrm{mL}$ and $0.24 \pm 0.01 \mu \mathrm{g} / \mathrm{mL}$, respectively. The result indicated that the $A U C_{I N F}$ and $C_{\text {max }}$ in ileum were significantly higher than that in plasma. Our results were consistent with previous study that APR displayed a much lower bioavailability and was beneficial for the treatment of gastrointestinal infections. Our result was also proved that the PK parameter in ileum was more accurate for PK/PD model of intestinal infection in pigs.

At present, the dosage of APR for the treatment of Salmonellosis has not been formulated. Clinical treatment always refers to the clinical recommended dose scheme of APR in the Chinese veterinary drug code. Wild type MIC distributions and $\mathrm{AUC}_{0-24 \mathrm{~h}} / \mathrm{MIC}$ ratios have a great effect on doses prediction. It is based on $\mathrm{MIC}_{90}$ for each pathogen and average values for other variables, only providing an initial indication of likely effective dosage, but does not take account either of variability or incidence of each input variable [27]. In our study, the MIC for 141 strains of total 185 strains was $8 \mu \mathrm{g} / \mathrm{mL}$, so whether the MIC 90 value can represent the actual clinical data remains to be further studied. In future studies, these concerns could be addressed by increasing numbers of isolates in field distribution studies and in PK/PD breakpoint estimation studies. The present study illustrates the principles of using MCS to predict dosages of APR for the treatment of Salmonellosis in the piglets. The proposed dosage regimen is for Salmonellosis induced by Salmonella only. For other organisms, independent PK/PD studies will be required.

$\mathrm{CBP}$ is used to determine whether the bacteria stain susceptibility or resistance. The $\mathrm{CO}_{\mathrm{WT}}, \mathrm{CO}_{\mathrm{PD}}$ and $\mathrm{CO}_{\mathrm{CL}}$ should be considered for the determination of $\mathrm{CBP}$ [14]. Previous studies showed that there was a gap existing in $\mathrm{CO}_{\mathrm{WT}}$ and $\mathrm{CO}_{\mathrm{CL}}$. It was reported that $\mathrm{CO} C \mathrm{CL}$ of danofloxacin against Glaesserella parasuis was $0.5 \mu \mathrm{g} / \mathrm{mL}$, but the $\mathrm{CO}_{\mathrm{WT}}$ of danofloxacin against Glaesserella parasuis was $8 \mu \mathrm{g} / \mathrm{mL}$ [28]. In our result, the $\mathrm{CO}_{\mathrm{CL}}$ of $\mathrm{APR}$ against Salmonella was $8 \mu \mathrm{g} / \mathrm{mL}$, but the $\mathrm{CO}_{\mathrm{WT}}$ of APR against Salmonella was $32 \mu \mathrm{g} / \mathrm{mL}$. So, our result was in accordance with the previous study. The possible reasons for the difference may be that the $\mathrm{CO}_{\mathrm{WT}}$ establishment was due to the epidemiological characteristic of the selected bacteria affected by geographical differences of strains, susceptibility of strains, etc.

Our study investigated variations in the composition and function of ileum microbiota during the diarrheic piglet treatment process. It was consistent with the results of previous studies that Firmicutes was the dominant phylum in the ileum of piglet, and there were no significant differences in relative abundance among groups $[14,18]$. The change of the abundances of Bacteroidetes and Euryarchaeota was the most obvious during the evolution process (Figure 6A). At the genus level, Methanobrevibacter, Prevotella, S24-7 and Ruminococcaceae were obtained as the highest abundance genus. The abundance of Methanobrevibacter 
increased significantly when APR treatment carried and decreased in cure and withdrawal period group. The abundance of Prevotella in test groups was significantly lower than that in the healthy group. A decreased of abundance in S24-7 was observed after Salmonella infection and increased slightly after cure. Ruminococcaceae increased significantly after Salmonella infection and decreased significantly after APR treatment (Figure 6B). The results indicated that the Salmonella infection and APR treatment had a great impact on the ileum flora evolution. An increase of abundance in Proteobacteria was previously obtained as a biomarker for intestinal microbial community dysbiosis and a potential diagnostic criterion for disease [29]. Interestingly, in this study, Proteobacteria and Lentisphaerae were described as the biomarker of diarrheic piglets by LEfSe software (Figure 7B). This was consistent the previous study and another biomarker was found in our results. A previous study showed that Prevotellacecea UCG-003 was a key node in diarrheic piglets upon co-correlation network analysis [30]. The genera of Methanobrevibacter and Prevotella was defined as the hub node by the Spearman network analysis; this result was different from previous study. The possible reason was that the sampling sites were different, and the composition of flora in ileum and piglet fecal were quite different. Another potential reason was the different effect of the selected challenge bacteria on the structure of flora. Further studies on the metabolites of the gut microbiome are needed to explore the correlation between these key genera and piglet diarrhea.

Antibiotics exert an effect on the amount and diversity of microbes causing change in functional diversity and colonization resistance against invading pathogens [31]. Previous study demonstrated that membrane transport, carbohydrate metabolism, amino acid metabolism, replication and repair, translation and energy metabolism were observed as the most abundant functions pathways by the prediction of microbial gene functions [32,33]. In this study, PICRUSt prediction revealed that the relative abundance of amino acid metabolism, carbohydrate metabolism, metabolism of cofactors and vitamins, metabolism of other amino acids, biosynthesis of other secondary metabolites, global and overview maps, replication and repair, glycan biosynthesis and metabolism, and lipid metabolism and energy metabolism. It was obvious that amino acid metabolism, replication and repair, and energy metabolism were obtained both in previous and in our results, so these KEGG pathways might play a key role in ileum flora evolution during the clinical treatment process.

\section{Materials and Methods}

\subsection{Chemicals}

APR sulfate Standard (purity of 98\%) was purchased from National Institutes for Food and Drug Control (Beijing, China). APR bulk (purity of $87.5 \%$ ) was provided by the Institute of Veterinary Pharmaceuticals (HZAU, Wuhan, China).

\subsection{Salmonella Isolates}

A total of 185 swine Salmonella strains were obtained from National Reference Laboratory of Veterinary Drug Residues (HZAU, Wuhan, China) and all the isolates were confirmed as the previous literature described [34].

\subsection{Animals}

Fifty-one four-week-old healthy crossbred (Duroc $\times$ Large $\times$ white $\times$ Landrace) pigs weighing $15 \pm 1 \mathrm{~kg}$ were purchased from Huazhong Agricultural University pig breeding farm. Eighty-one 7-week-old healthy female Kunming mice weighing $25 \pm 5 \mathrm{~g}$ were purchased from the Experimental Animal Center of Wuhan University. Prior to experiments, piglets and mice were raised for 7 days to acclimatize. All the animal experiments were approved by the Animal Ethics Committee of Huazhong Agricultural University (hzauch 2014-003) and the Animal Care Center, Hubei Science and Technology Agency in China (SYXK2013-0044). All animal experiments were conducted according to the committee guidelines for the Laboratory Animal Use and Care Committee in Hubei Science 
and Technology Agency. All efforts were used to reduce the pain and adverse effect of the animals.

\subsection{Establishment of $\mathrm{CO}_{W T}$}

The susceptibility of 185 swine Salmonella strains to APR was determined in triplicate by broth micro-dilution method according to Clinical and Laboratory Institute guidelines [35]. The MIC distribution was constructed as previously described [36]. The final ECOFF was measured as that MIC which captured at least $95 \%$ of the optimum MIC distributions calculated by the ECOFFinder software [37].

\subsection{Model of Salmonella Infection}

Thirteen isolates carrying more virulence genes was confirmed by multiplex Polymerase Chain Reaction (PCR); the primers and procedure are presented in Tables S9 and S10. Then, a model of Salmonella infection was established as follows. Briefly, female Kunming mice were infected at the age of 8 weeks. Food and water were provided ad libitum. Mice were inoculated by intraperitoneal infection with $200 \mu \mathrm{L}$ of inoculum containing a total of $2 \times 10^{7}$ or $2 \times 10^{9}$ bacteria with three mice in each dose group. The control group was inoculated by intraperitoneal infection with $200 \mu \mathrm{L}$ Luria-Bertan (LB) broth. The clinical symptoms of mice after Salmonella challenge were observed, and the target bacteria were isolated and identified after anatomical examination of poisoning mouse. Eventually, strains $3303,10 \#, 34 \#, 10,643$ and 5023, with an MIC of 4, 8, 16, 32 and $256 \mu \mathrm{g} / \mathrm{mL}$, respectively, were selected for the further experiment.

\subsection{PD Study In Vitro and Ex Vivo}

The MIC, MBC and MPC of APR against Salmonella 10\# were determined in broth plasma and ileum fluid. PAE of APR against swine Salmonella was determined with the removal of drug methods. Strain 10\# was exposed to $1 \times$ MIC, $2 \times$ MIC, $4 \times$ MIC of APR. At 1 and $2 \mathrm{~h}$, the removal drug culture was obtained by centrifuging and was re-cultured in fresh MH broth without APR. The colony-forming units (CFU) were determined at a series time-point.

The in vitro and ex vivo killing curves of APR in broth and in ileum fluid were drawn by monitoring the CFU changes during the incubation of Salmonella 10\# under a series concentration of APR (1/2 to $32 \mathrm{MIC})$ for a continuous time period $(0,1,2,4,6,8,12$ and $24 \mathrm{~h})[28]$.

\subsection{Samples Collection for PK Study}

Twelve piglets were divided randomly into two groups with equal numbers of males and females in each group. Each group was treated via the intragastric route with a single dose of $30 \mathrm{mg} / \mathrm{kg}$ b.w. After administration, $2 \mathrm{~mL}$ blood samples were obtained at $0,0.5$, $1,2,3,4,6,8,10,12,24$ and $48 \mathrm{~h}$. Plasma was obtained followed by centrifugation at $3500 \mathrm{r} / \mathrm{min}$ for $10 \mathrm{~min}$ at $4{ }^{\circ} \mathrm{C}$. Ileum contents $(5 \mathrm{~g})$ from each pig were gently collected at time-point as that of the plasma. All the samples were stored at $-80^{\circ} \mathrm{C}$ prior to the analysis.

\subsection{Samples Analysis for PK}

The APR concentration in serum and ileum was determined using a high-performance liquid chromatography-tandem mass spectrometry (HPLC-MS/MS) method. In brief, serum samples $(0.5 \mathrm{~mL})$ and ileum contents $(1 \mathrm{~g})$ were treated with three times the volume of acetonitrile, vibrated for $30 \mathrm{~s}$, and centrifuged at 14,000 r/min for $5 \mathrm{~min}$. Then volume of $400 \mu \mathrm{L} 4 \% \mathrm{H}_{3} \mathrm{PO}_{4}$ was added to equal volume of supernatant to adjust the $\mathrm{pH}$. An Oasis HLB 60-mg extraction cartridge (Waters) was successively conditioned with 3-5 $\mathrm{mL}$ of methanol before equilibrated with $3-5 \mathrm{~mL}$ of $\mathrm{H}_{2} \mathrm{O}$. The cartridge was then rinsed with $0.8 \mathrm{~mL}$ of $5 \%$ methanol aqueous solution and dried under nitrogen for $4 \mathrm{~min}$. Compounds of interest were eluted with $50 \mu \mathrm{L}$ acetonitrile-methanol $(50: 25, v / v)$. The eluate was evaporated to dryness under a nitrogen stream at $30{ }^{\circ} \mathrm{C}$, and the residue was 
taken up with $1 \mathrm{~mL} 0.1 \%$ formic acid in ammonium acetate-acetonitrile $(15: 85, v / v)$ before HPLC-MS-MS injection.

Ten microliters of each sample was injected onto a Shimadzu 30A system (Shimadzu, Japan), which included a binary pump, refrigerated autosampler $\left(4^{\circ} \mathrm{C}\right)$ and a temperaturecontrolled column compartment $\left(30^{\circ} \mathrm{C}\right)$. Chromatographic separation was performed using a Waters Acquity BEH C18 column $(2.1 \times 100 \mathrm{~mm}, 1.7 \mu \mathrm{m}$, Waters, Milford, MA, USA). The mobile phase consisted of A: $0.1 \%$ formic acid in ammonium acetate, and B: acetonitrile with a flow rate of $0.2 \mathrm{~mL} / \mathrm{min}$ and a total run time of $4 \mathrm{~min}$. A gradient of mobile phase B was utilized by decreasing the initial $85 \%$ linearly to $10 \%$ over $0.4 \mathrm{~min}$, held there until $2.3 \mathrm{~min}$, then returned to $85 \%$ by $2.4 \mathrm{~min}$.

Detection of the APR was performed by an AB SCIEX QTRAP ${ }^{\circledR} 5500$ mass spectrometer (AB SCIEX, Framingham, MA, USA). The mass spectrometer was operated to monitor the monoisotopes of APR $(\mathrm{m} / \mathrm{z} 540.3 \rightarrow 378.4 \rightarrow 344.4 \rightarrow 217.4)$ using multiple reaction monitoring (MRM) under positive ionization mode. The ion spray voltage was set at $5500 \mathrm{~V}$ for positive monitoring, and the temperature was maintained at $550{ }^{\circ} \mathrm{C}$. The nebulizing gas was high-purity nitrogen, and atomization gas was 65 psi, auxiliary gas was 60 psi and air curtain gas was 30 psi. Data processing was performed using the MultiQuant ${ }^{\mathrm{TM}}$ software (Version 3.0.3, AB SCIEX). The concentration-time data for APR in plasma and ileum samples harvested from healthy/infection piglets were analyzed by WinNonlin software (Version 6.3; Pharsight Inc., St. Louis, MO, USA) to obtain the pharmacokinetic parameters.

\subsection{PK and PD Analyses, Dosage Prediction and Establishment of $C O_{P D}$}

To determine the PK/PD integration of APR in the ileum fluid, the $\mathrm{AUC}_{0-24 \mathrm{~h}} / \mathrm{MIC}$ was calculated according to $\mathrm{PK}$ data and ex vivo $\mathrm{PD}$ data. Changes in bacterial abundance in ileum were integrated with the PK/PD parameters $\mathrm{AUC}_{0-24 \mathrm{~h}} / \mathrm{MIC}$ using the sigmoid $\mathrm{E}_{\max }$ model with WinNonlin as the previously described [27]. Based on the results of PK-PD modeling for the relationship between $\mathrm{AUC}_{0-24 \mathrm{~h}} / \mathrm{MIC}$ and the ex vivo antibacterial effect, three levels of antibacterial effect of APR were quantified from the sigmoid $\mathrm{E}_{\max }$ equation by determining $\mathrm{AUC}_{0-24 \mathrm{~h}} / \mathrm{MIC}$ required for bacteriostatic action as previously described [38]. PK/PD parameter values corresponding to the $\mathrm{E}$ value (derived from the sigmoid $\mathrm{E}_{\max }$ equation) in ileal fluid were used to deduce an optimal dose regimen [39]. The PD target was selected to calculate the probability of PTA. COPD was defined as the MIC at which the PTA was $\geq 90 \%$ under 10,000 trials with Crystal Ball software (version 7.2.2, Oracle, Austin, TX, USA) [39].

\subsection{Infection Model and Clinical Trials}

Thirty-nine healthy weaned piglets with body weight of $(15 \pm 1 \mathrm{~kg})$ were divided into seven groups including one blank control group (3 piglets), one positive control group infected without APR treatment (6 piglets) and five infected groups treated with APR (each 6 piglets), to obtain clinical data for APR in the treatment of Salmonella infection. Each piglet in the infected group was inoculated by intragastric administration with $50 \mathrm{~mL}$ of $1 \times 10^{10} \mathrm{CFU} / \mathrm{mL}$ Salmonella strains 3303, 10\#, 34\#, 10,643 and 5023, two times per day and per piglet for three consecutive days. The blank control group was inoculated with blank LB broth. The dosage regimens were recommended by the PK-PD therapeutic dosage regimen. After challenged, these pigs were monitored daily for 4 days.

\subsection{Statistical Analysis for Establishment of $\mathrm{CO}_{C L}$}

Simply, the data was firstly analyzed by the "WindoW" approach to obtain two parameters: "MaxDiff" (the method of maximum difference) and "CAR" (the curve (AUC) ratio) as the previously described [14]. According to the formula POC $=1 /\left(1+\mathrm{e}^{-\mathrm{a}+\mathrm{bf}(\mathrm{MIC})}\right)$ proposed by EUCAST, the obtained data in the "WindoW" approach was fitted by the nonlinear regression analysis using SPSS software. Then the MIC value corresponding to the cure rate of $90 \%$ was calculated. Eventually, CART software (version 6.0; Salford Systems, San Diego, CA, USA) was used for the establishment of $\mathrm{CO}_{\mathrm{CL}}$ [14]. 


\subsection{Samples Collection of Gut Flora $16 S$ rRNA Gene Sequencing}

Ileal contents samples were collected from the group infected by Salmonella strains 10\# including five time nodes of before infection, infection, APR treatment, cure and withdrawal, sequentially named as the healthy group (GY), the infection group (GY0), the treatment group (GY24), the cure group (GY72) and the withdrawal period group (GY96). Except for 3 samples collected in the cure group, only 2 samples were collected at other times of sampling. Then content samples were snap-frozen in liquid nitrogen and stored at $-80^{\circ} \mathrm{C}$.

\subsection{3. $16 S$ rRNA Gene Sequencing}

In short, microbial genomic DNA was extracted from $200 \mathrm{mg}$ of ileum content samples using the TIANGEN DNA stool mini kit (TIANGEN, Beijing, China) following the manufacturer's guidelines. Then, the $16 S$ rRNA gene of the V3-V4 region was amplified using specific primers as previously described [40]. The resulting PCR products were extracted from a $2 \%$ agarose gel and further purified using a TIANGEN gel DNA extraction kit (TIANGEN, Beijing, China). Purified PCR products were sequenced on an Illumina MiSeq platform by Bioacme Biotechnology Co., Ltd. (Shanghai, China). The raw data are available in the NCBI SRA database under BioProject accession number PRJNA761542.

\subsection{Analysis of Microbial Diversity}

To obtain high-quality clean tags, quality filtering of the raw tags was performed using the Quantitative Insights Into Microbial Ecology software (QIIME, version 1.9.1) qualitycontrolled process [41]. The chimaeric sequences were removed by using the UCHIME Algorithm and then the effective tags were obtained [42]. The sequences were clustered into OTUs at $97 \%$ similarity by UPARSE software (version 7.0.1001) [43]. In addition, the GreenGene database and MUSCLE software (version 3.8.31) were used to annotate the taxonomic information and conduct the multiple sequence alignment, respectively [44]. The alpha diversity index (Chao1, ACE, Shannon, Simpson indices), the beta diversity index, PCoA, NMDS and ANOSIM were conducted as previously described [45].

\subsection{Microbial Taxonomic Analysis}

To identify the genomic features of taxa differing in abundance between two or more biological conditions or classes, the LEfSe algorithm was used with the online interface Galaxy (http:/ / huttenhower.sph.harvard.edu/lefse/, accessed on 4 September 2021) [46]. A size-effect threshold of 4.0 on the logarithmic LDA score was used for discriminative functional biomarkers. A Phylogenetic Investigation of Communities by Reconstruction of Unobserved States (PICRUSt, version 1.0.0) was performed to predict the ileal microbial community shift of the five groups of piglets. According to the calculation method described in the previous study [47], co-correlation networks were generated using the MENA (http:/ /ieg4.rccc.ou.edu/MENA/, accessed on 1 September 2021) and the OTUs as target nodes, with edges (e.g., connecting nodes) representing significant negative (green) or positive (red) Spearman's correlations. We retained OTUs when they had a Spearman's correlation coefficient $>0.5$. The predicted genes and their function were aligned to KEGG database and the differences among groups were compared through software STAMP (version 2.1.3) [48].

\section{Conclusions}

This study firstly established the $\mathrm{CO}_{\mathrm{WT}}, \mathrm{CO}_{\mathrm{PD}}$ in ileum and $\mathrm{CO}_{\mathrm{CL}}$ of APR against swine Salmonella, the value of these cutoffs were $32 \mu \mathrm{g} / \mathrm{mL}, 32 \mu \mathrm{g} / \mathrm{mL}$ and $8 \mu \mathrm{g} / \mathrm{mL}$, respectively. According to the guiding principle of the CLSI, the final CBP in ileum was $32 \mu \mathrm{g} / \mathrm{mL}$. Our results revealed the main evolution route in the composition of ileum microbiota of diarrheic piglets treated by APR. The change of the abundances of Bacteroidetes and Euryarchaeota was the most obvious during the evolution process. Methanobrevibacter, Prevotella, S24-7 and Ruminococcaceae were obtained as the highest abundance genus. The 
abundance of Methanobrevibacter increased significantly when APR treatment carried and decreased in the cure and withdrawal period groups. The abundance of Prevotella in test groups was significantly lower than that in the healthy group. A decreased of abundance in S24-7 was observed after Salmonella infection and increased slightly after cure. Ruminococcaceae increased significantly after Salmonella infection and decreased significantly after APR treatment. In addition, the genera of Methanobrevibacter and Prevotella were defined as the key node. Valine, leucine and isoleucine biosynthesis, D-Glutamine and D-glutamate metabolism, D-Alanine metabolism, peptidoglycan biosynthesis and biosynthesis of amino acids were the top five KEGG pathways in the ileum microbiota of piglets during the Salmonella infection and APR treatment process.

Supplementary Materials: The following are available online at https:/ / www.mdpi.com/article/10 $.3390 /$ ijms23031424/s1.

Author Contributions: Conceptualization, H.H. and X.D.; methodology, H.H., J.G., L.H. and X.D.; software, Y.G.; validation, J.G., X.D. and H.H.; formal analysis, X.D. and D.P.; investigation, X.D., G.C. and D.P.; resources, X.D.; data curation, L.H. and G.C.; writing-original draft preparation, Y.G.; writing-review and editing, Y.G. and H.H.; visualization, Y.G.; supervision, H.H. and D.P.; project administration, H.H.; funding acquisition, H.H. All authors have read and agreed to the published version of the manuscript.

Funding: This work was funded by National Natural Science Foundation of China (31772791/32172914), The National Key Research and Development Program (2021YFD1800600/2016YFD0501302), Fundamental Research Funds for the Central Universities (2662018JC001).

Institutional Review Board Statement: All the animal experiments were approved by the Animal Ethics Committee of Huazhong Agricultural University (hzauch 2014-003) and the Animal Care Center, Hubei Science and Technology Agency in China (SYXK2013-0044). All animal experiments were conducted according to the committee guidelines for the Laboratory Animal Use and Care Committee in Hubei Science and Technology Agency. All efforts were used to reduce the pain and adverse effect of the animals.

Informed Consent Statement: Not applicable.

Data Availability Statement: The sequencing data was submitted to the National Center for Biotechnology Information Sequence Read Archive (SRA) under Accession No. PRJNA761542.

Conflicts of Interest: The authors declare that the research was conducted in the absence of any commercial or financial relationships that could be construed as a potential conflict of interest.

\section{References}

1. Garrido, V.; Sánchez, S.; Román, B.S.; Fraile, L.; Migura, L.; Grilló, M.-J. Salmonella Infection in Mesenteric Lymph Nodes of Breeding Sows. Foodborne Pathog. Dis. 2019, 17, 411-417. [CrossRef] [PubMed]

2. Besser, J.M. Salmonella epidemiology: A whirlwind of change. Food Microbiol. 2018, 71, 55-59. [CrossRef] [PubMed]

3. Forge, A.; Schacht, J. Aminoglycoside antibiotics. Audiol. Neurotol. 2004, 5, 3-22. [CrossRef] [PubMed]

4. Shi, K.; Caldwell, S.J.; Fong, D.H.; Berghuis, A.M. Prospects for circumventing aminoglycoside kinase mediated antibiotic resistance. Front. Cell. Infect. Microbiol. 2013, 3, 22. [CrossRef] [PubMed]

5. O'Connor, S.; Lam, L.K.T.; Jones, N.D.; Chaney, M.O. Apramycin, a unique aminocyclitol antibiotic. J. Org. Chem. 1976, 41, 2087-2092. [CrossRef]

6. Ramirez, M.S.; Tolmasky, M.E. Aminoglycoside modifying enzymes. Drug Resist. Updat. 2010, 13, 151-171. [CrossRef] [PubMed]

7. Antunes, E.D.A.B.; Lourenço, F.R.; Pinto, T.D.J.A. Determination of apramycin in oral soluble powder by a HPLC method using pre-column derivatization with o-phthalaldehyde and UV detection. Braz. J. Pharm. Sci. 2011, 47, 261-268. [CrossRef]

8. Bischoff, K.; Edrington, T.; Callaway, T.; Genovese, K.; Nisbet, D. Characterization of antimicrobial resistant Salmonella Kinshasa from dairy calves in Texas. Lett. Appl. Microbiol. 2004, 38, 140-145. [CrossRef]

9. Choi, M.-J.; Lim, S.-K.; Nam, H.-M.; Kim, A.-R.; Jung, S.-C.; Kim, M.-N. Apramycin and gentamicin resistances in indicator and clinical escherichia coli isolates from farm animals in Korea. Foodborne Pathog. Dis. 2011, 8, 119-123. [CrossRef]

10. Yates, C.M.; Pearce, M.C.; Woolhouse, M.E.J.; Amyes, S.G.B. High frequency transfer and horizontal spread of apramycin resistance in calf faecal Escherichia coli. J. Antimicrob. Chemother. 2004, 54, 534-537. [CrossRef] 
11. Jensen, V.F.; Jakobsen, L.; Emborg, H.-D.; Seyfarth, A.M.; Hammerum, A.M. Correlation between apramycin and gentamicin use in pigs and an increasing reservoir of gentamicin-resistant Escherichia coli. J. Antimicrob. Chemother. 2006, 58, 101-107. [CrossRef] [PubMed]

12. Toutain, P.-L.; Bousquet-Melou, A.; Damborg, P.; Ferran, A.A.; Mevius, D.; Pelligand, L.; Veldman, K.T.; Lees, P. En route towards European clinical breakpoints for veterinary antimicrobial susceptibility testing: A position paper explaining the vetcast approach. Front. Microbiol. 2017, 8, 2344. [CrossRef] [PubMed]

13. Schuetz, A.N. Role of bacterial and fungal epidemiological cutoff values for antimicrobial susceptibility testing in the microbiology laboratory. Clin. Microbiol. Newsl. 2020, 42, 9-17. [CrossRef]

14. Turnidge, J.D.; Martinez, M.N. Proposed method for estimating clinical cut-off (CO CL ) values: An attempt to address challenges encountered when setting clinical breakpoints for veterinary antimicrobial agents. Vet. J. 2017, 228, 33-37. [CrossRef]

15. Yatsunenko, T.; Rey, F.E.; Manary, M.J.; Trehan, I.; Dominguez-Bello, M.G.; Contreras, M.; Magris, M.; Hidalgo, G.; Baldassano, R.N.; Anokhin, A.P.; et al. Human gut microbiome viewed across age and geography. Nature 2012, 486, 222-227. [CrossRef]

16. Ashida, H.; Ogawa, M.; Kim, M.; Mimuro, H.; Sasakawa, C. Bacteria and host interactions in the gut epithelial barrier. Nat. Chem. Biol. 2012, 8, 36-45. [CrossRef] [PubMed]

17. Bauer, E.; Williams, B.A.; Smidt, H.; Verstegen, M.W.; Mosenthin, R. Influence of the gastrointestinal microbiota on development of the immune system in young animals. Curr. Issues Intest. Microbiol. 2006, 7, 35-42.

18. Sun, J.; Du, L.; Li, X.; Zhong, H.; Ding, Y.; Liu, Z.; Ge, L. Identification of the core bacteria in rectums of diarrheic and non-diarrheic piglets. Sci. Rep. 2019, 9, 18675. [CrossRef] [PubMed]

19. Kang, A.D.; Smith, K.P.; Eliopoulos, G.M.; Berg, A.H.; McCoy, C.; Kirby, J.E. In vitro apramycin activity against multidrug-resistant Acinetobacter baumannii and Pseudomonas aeruginosa. Diagn. Microbiol. Infect. Dis. 2017, 88, 188-191. [CrossRef]

20. Randall, L.; Ridley, A.; Lemma, F.; Hale, C.; Davies, R. In vitro investigations into the use of antimicrobials in combination to maintain efficacy of fluoroquinolones in poultry. Res. Vet. Sci. 2016, 108, 47-53. [CrossRef]

21. Tian, E.; Muhammad, I.; Hu, W.; Wu, Z.; Li, R.; Lu, X.; Chen, C.; Li, J. Tentative epidemiologic cut-off value and resistant characteristic detection of apramycin against Escherichia coli from chickens. FEMS Microbiol. Lett. 2019, 16, 16. [CrossRef] [PubMed]

22. Theuretzbacher, U. Tissue penetration of antibacterial agents: How should this be incorporated into pharmacodynamic analyses? Curr. Opin. Pharmacol. 2007, 7, 498-504. [CrossRef] [PubMed]

23. Zhao, M.; Lepak, A.J.; Andes, D.R. Animal models in the pharmacokinetic/pharmacodynamic evaluation of antimicrobial agents Bioorg. Med. Chem. 2016, 24, 6390-6400. [CrossRef] [PubMed]

24. Dai, C.; Zhao, T.; Yang, X.; Xiao, X.; Velkov, T.; Tang, S. Pharmacokinetics and relative bioavailability of an oral amoxicillinapramycin combination in pigs. PLoS ONE 2017, 12, e0176149. [CrossRef]

25. Shang, R.F.; Hu, Z.Y.; Zhang, X.G.; Li, S.H.; Shi, Y.B.; Xu, Z.Z.; Song, Z.S. Pharmacokinetics and bioavailability of Apramycin sulfate by oral administration in pigs. Chin. J. Vet. Med. 2004, 40, 3-5.

26. Afifi, N.; Ramadan, A. Kinetic disposition, systemic bioavailability and tissue distribution of apramycin in broiler chickens. Res. Vet. Sci. 1997, 62, 249-252. [CrossRef]

27. Dorey, L.; Pelligand, L.; Lees, P. Prediction of marbofloxacin dosage for the pig pneumonia pathogens Actinobacillus pleuropneumoniae and Pasteurella multocida by pharmacokinetic/pharmacodynamic modelling. BMC Vet. Res. 2017, 13, 209. [CrossRef]

28. Xu, Z.; Huang, A.; Luo, X.; Zhang, P.; Huang, L.; Wang, X.; Mi, K.; Fang, S.; Huang, X.; Li, J.; et al. Exploration of Clinical Breakpoint of Danofloxacin for Glaesserella parasuis in Plasma and in PELF. Antibiotics 2021, 10, 808. [CrossRef]

29. Looft, T.; Johnson, T.A.; Allen, H.K.; Bayles, D.O.; Alt, D.P.; Stedtfeld, R.D.; Sul, W.J.; Stedtfeld, T.M.; Chai, B.; Cole, J.R.; et al. In-feed antibiotic effects on the swine intestinal microbiome. Proc. Natl. Acad. Sci. USA 2012, 109, 1691-1696. [CrossRef]

30. Shin, N.-R.; Whon, T.W.; Bae, J.-W. Proteobacteria: Microbial signature of dysbiosis in gut microbiota. Trends Biotechnol. 2015, 33, 496-503. [CrossRef]

31. Ferrer, M.; Méndez-García, C.; Rojo, D.; Barbas, C.; Moya, A. Antibiotic use and microbiome function. Biochem. Pharmacol. 2016, 134, 114-126. [CrossRef]

32. Li, Y.; Zhu, Y.; Wei, H.; Chen, Y.; Shang, H. Study on the diversity and function of gut microbiota in pigs following long-term antibiotic and antibiotic-free breeding. Curr. Microbiol. 2020, 77, 4114-4128. [CrossRef] [PubMed]

33. Li, N.; Huang, S.; Jiang, L.; Dai, Z.; Li, T.; Han, D.; Wang, J. Characterization of the early life microbiota development and predominant lactobacillus species at distinct gut segments of low- and normal-birth-weight piglets. Front. Microbiol. 2019, 10, 797. [CrossRef]

34. Rodríguez-Hernández, R.; Bernal, J.; Cifuentes, J.; Fandiño, L.; Herrera-Sánchez, M.; Rondón-Barragán, I.; Garcia, N.V. Prevalence and molecular characterization of Salmonella isolated from broiler farms at the Tolima Region-Colombia. Animals 2021, 11, 970. [CrossRef] [PubMed]

35. CLSI. Performance Standards for Antimicrobial Susceptibility Testing: 28th Informational Supplement; CLSI document M100-S28; Clinical and Laboratory Standards Institute: Wayne, PA, USA, 2018.

36. Yang, Y.; Zhang, Y.; Li, J.; Cheng, P.; Xiao, T.; Muhammad, I.; Yu, H.; Liu, R.; Zhang, X. Susceptibility breakpoint for Danofloxacin against swine Escherichia coli. BMC Vet. Res. 2019, 15, 51. [CrossRef] [PubMed] 
37. Turnidge, J.; Kahlmeter, G.; Kronvall, G. Statistical characterisation of bacterial wild-type MIC value distributions and the determination of epidemiological cut-off values. Clin. Microbiol. Infect. 2010, 12, 418-425. [CrossRef]

38. Wang, F.; Mi, K.; Ahmad, I.; Xie, S.; Hussain, H.I.; Yuan, Z.; Dai, M.; Huang, L. Antibacterial activity of cyadox against Clostridium perfringens in broilers and a dosage regimen design based on pharmacokinetic-pharmacodynamic modeling. Microb. Pathog. 2020, 141, 103981. [CrossRef]

39. Zhou, Y.-F.; Bu, M.-X.; Liu, P.; Sun, J.; Liu, Y.-H.; Liao, X.-P. Epidemiological and PK/PD cutoff values determination and PK/PD-based dose assessment of gamithromycin against Haemophilus parasuis in piglets. BMC Vet. Res. 2020, 16, 81. [CrossRef]

40. Yang, Y.; Liu, Y.; Liu, J.; Wang, H.; Guo, Y.; Du, M.; Cai, C.; Zhao, Y.; Lu, C.; Guo, X.; et al. Composition of the fecal microbiota of piglets at various growth stages. Front. Vet. Sci. 2021, 8, 661-671. [CrossRef]

41. Caporaso, J.G.; Kuczynski, J.; Stombaugh, J.; Bittinger, K.; Bushman, F.D.; Costello, E.K.; Fierer, N.; Peña, A.G.; Goodrich, J.K.; Gordon, J.I.; et al. QIIME allows analysis of high-throughput community sequencing data. Nat. Methods 2010, 7, 335-336. [CrossRef] [PubMed]

42. Edgar, R.C.; Haas, B.J.; Clemente, J.C.; Quince, C.; Knight, R. UCHIME improves sensitivity and speed of chimera detection. Bioinformatics 2011, 27, 2194-2200. [CrossRef]

43. Edgar, R.C. UPARSE: Highly accurate OTU sequences from microbial amplicon reads. Nat. Methods 2013, 10, 996-998. [CrossRef] [PubMed]

44. Toth, F.; Frank, N.; Elliott, S.B.; Geor, R.; Boston, R.C. Effects of an intravenous endotoxin challenge on glucose and insulin dynamics in horses. Am. J. Vet. Res. 2004, 69, 82-88. [CrossRef] [PubMed]

45. Xu, P.; Shi, Y.; Liu, P.; Yang, Y.; Zhou, C.; Li, G.; Luo, J.; Zhang, C.; Cao, H.; Hu, G.; et al. $16 \mathrm{~S}$ rRNA gene sequencing reveals an altered composition of the gut microbiota in chickens infected with a nephropathogenic infectious bronchitis virus. Sci. Rep. 2020, 10, 3556. [CrossRef] [PubMed]

46. Segata, N.; Izard, J.; Waldron, L.; Gevers, D.; Miropolsky, L.; Garrett, W.S.; Huttenhower, C. Metagenomic biomarker discovery and explanation. Genome Biol. 2011, 12, R60. [CrossRef]

47. Deng, Y.; Jiang, Y.-H.; Yang, Y.; He, Z.; Luo, F.; Zhou, J. Molecular ecological network analyses. BMC Bioinform. $2012,13,113$. [CrossRef]

48. Parks, D.H.; Beiko, R.G. Identifying biologically relevant differences between metagenomic communities. Bioinformatics 2010, 26, 715-721. [CrossRef] 